Bull. Korean Math. Soc. 50 (2013), No. 6, pp. 2061-2070

http://dx.doi.org/10.4134/BKMS.2013.50.6.2061

\title{
GENERALIZED HYERS-ULAM-RASSIAS STABILITY FOR A GENERAL ADDITIVE FUNCTIONAL EQUATION IN QUASI- $\beta$-NORMED SPACES
}

\author{
Fridoun Moradlou and Themistocles M. Rassias
}

\begin{abstract}
In this paper, we investigate the generalized HyersUlamRassias stability of the following additive functional equation

$$
2 \sum_{j=1}^{n} f\left(\frac{x_{j}}{2}+\sum_{i=1, i \neq j}^{n} x_{i}\right)+\sum_{j=1}^{n} f\left(x_{j}\right)=2 n f\left(\sum_{j=1}^{n} x_{j}\right)
$$
\end{abstract}

in quasi- $\beta$-normed spaces.

\section{Introduction and preliminaries}

A classical question in the theory of functional equations is the following: "When is it true that a function, which approximately satisfies a functional equation $\mathcal{E}$ must be close to an exact solution of $\mathcal{E}$ ?" If the problem accepts a solution, we say that the equation $\mathcal{E}$ is stable. Such a problem was formulated by Ulam [42] in 1940 and solved in the next year for the Cauchy functional equation by Hyers [12]. It gave rise the stability theory for functional equations. The result of Hyers was extended by Aoki [2] in 1950, by considering the unbounded Cauchy differences. In 1978, Th. M. Rassias [36] proved that the additive mapping $T$, obtained by Hyers or Aoki, is linear if, in addition, for each $x \in E$ the mapping $f(t x)$ is continuous in $t \in \mathbb{R}$. Găvruta [10] generalized the Rassias' result. Following the techniques of the proof of the corollary of Hyers [12] we observed that Hyers introduced (in 1941) the following Hyers continuity condition: about the continuity of the mapping for each fixed, and then he proved homogenouity of degree one and therefore the famous linearity. Beginning around the year 1980, the stability problems of several functional equations and approximate homomorphisms have been extensively investigated by a number of authors and there are many interesting results concerning this problem (see [1], [5], [8], [14]-[29], [32]-[40]).

Received January 2, 2013.

2010 Mathematics Subject Classification. Primary 39B72, 39B82, 46B03, 47Jxx.

Key words and phrases. generalized Hyers-Ulam stability, contractively subadditive, expansively superadditive, quasi- $\beta$-normed space, $(\beta, p)$-Banach space. 
In 2003 Cădariu and Radu applied the fixed point method to the investigation of the Jensen functional equation [6] (see also [7], [8], [16], [30]). They could present a short and a simple proof (different of the "direct method", initiated by Hyers in 1941) for the generalized Hyers-Ulam stability of Jensen functional equation [6], for Cauchy functional equation [8] and for quadratic functional equation [7].

The following functional equation

$$
Q(x+y)+Q(x-y)=2 Q(x)+2 Q(y)
$$

is called a quadratic functional equation, and every solution of equation (1.1) is said to be a quadratic mapping. F. Skof [41] proved the Hyers-Ulam stability of the quadratic functional equation (1.1) for mappings $f: E_{1} \rightarrow E_{2}$, where $E_{1}$ is a normed space and $E_{2}$ is a Banach space. In [9], S. Czerwik proved the Hyers-Ulam stability of the quadratic functional equation (1.1). C. Borelli and G. L. Forti [4] generalized the stability result of the quadratic functional equation (1.1).

Recently, P. Găvruta and L. Găvruta used a new method for investigation of Hyers-Ulam-Rassias stability of a nonlinear functional equation, Volterra integral operator and Fredholm operator. This method generalized the fixed point method [11].

We consider some basic concepts concerning quasi- $\beta$-normed spaces and some preliminary results. We fix a real number $\beta$ with $0<\beta \leq 1$ and let $\mathbb{K}$ denote either $\mathbb{R}$ or $\mathbb{C}$. Let $X$ be a linear space over $\mathbb{K}$. A quasi- $\beta$-norm $\|\cdot\|$ is a real-valued function on $X$ satisfying the following:

(i) $\|x\| \geq 0$ for all $x \in X$ and $\|x\|=0$ if and only if $x=0$.

(ii) $\|\lambda x\|=|\lambda|^{\beta}\|x\|$ for all $\lambda \in \mathbb{K}$ and all $x \in X$.

(iii) There is a constant $K \geq 1$ such that $\|x+y\| \leq K(\|x\|+\|y\|)$ for all $x, y \in X$.

The pair $(X,\|\cdot\|)$ is called a quasi- $\beta$-normed space if $\|\cdot\|$ is a quasi- $\beta$-norm on $\mathrm{X}$. The smallest possible $K$ is called the modulus of concavity of $\|\cdot\|$. A quasi- $\beta$-Banach space is a complete quasi- $\beta$-normed space.

A quasi- $\beta$-norm $\|\cdot\|$ is called a $(\beta, p)$-norm $(0<p \leq 1)$ if

$$
\|x+y\|^{p} \leq\|x\|^{p}+\|y\|^{p}
$$

for all $x, y \in X$. In this case, a quasi- $\beta$-Banach space is called a $(\beta, p)$-Banach space.

By the Aoki-Rolewicz theorem [40] (see also [3]), each quasi-norm is equivalent to some $p$-norm. Since it is much easier to work with $p$-norms than quasi-norms, henceforth we restrict our attention mainly to $p$-norms.

Example 1.1. For $x=\left(x_{1}, x_{2}\right) \in \mathbb{R}^{2}$, we define

$$
\|x\|_{p, \beta}=\left\{\begin{array}{cl}
\left(\left|x_{1}\right|^{\beta p}+\left|x_{2}\right|^{\beta p}\right)^{\frac{1}{p}} ; & x_{2} \neq 0 \\
2\left|x_{1}\right|^{\beta} ; & x_{2}=0
\end{array}\right.
$$


where $0<p, \beta \leq 1$. Then $\left(\mathbb{R}^{2},\|x\|_{p, \beta}\right)$ is a $(\beta, p)$-norm space.

Example 1.2. If $X$ is a quasi- $\beta$-norm space with the quasi- $\beta$-norm $\|x\|_{\beta}$, then it is a quasi-norm space with the quasi-norm $\|x\|=\|x\|_{\beta}^{\frac{1}{\beta}}$.

In this paper, for a fixed positive integer $n \geq 2$, we introduce the following additive functional equation of Cuachy-Jensen type:

$$
2 \sum_{j=1}^{n} f\left(\frac{x_{j}}{2}+\sum_{i=1, i \neq j}^{n} x_{i}\right)+\sum_{j=1}^{n} f\left(x_{j}\right)=2 n f\left(\sum_{j=1}^{n} x_{j}\right) .
$$

It is easy to see that the function $f(x)=a x$ is a solution of the functional equation (1.2). Every solution of the functional equation (1.2) is said to be a generalized additive mapping of Cauchy-Jensen type.

Throughout this paper, $n$ will be a positive integer such that $n \geq 2$.

\section{Generalized Hyers-Ulam-Rassias stability of Eq.(1.2): fixed point method}

In this section, by using the idea of P. Găvruta and L. Găvruta [11], we prove the generalized Hyers-Ulam-Rassias stability of Eq.(1.2) in $(\beta, p)$-Banach spaces.

Lemma 2.1. Let $\mathcal{X}$ and $\mathcal{Y}$ be linear spaces and suppose that a mapping $L: \mathcal{X} \rightarrow \mathcal{Y}$ satisfies the functional equation (1.2) for all $x_{1}, \ldots, x_{n} \in \mathcal{X}$. Then the mapping $L$ is Cauchy additive.

Proof. Putting $x_{1}=\cdots=x_{n}=0$ in (1.2), we get $L(0)=0$. Letting $x_{m}=0$ in (1.2) for all $1 \leq m \leq n$ with $m \neq i, j$, we get

$$
2 L\left(\frac{x_{i}}{2}+x_{j}\right)+2 L\left(\frac{x_{j}}{2}+x_{i}\right)+L\left(x_{i}\right)+L\left(x_{j}\right)=4 L\left(x_{i}+x_{j}\right)
$$

for all $x_{i}, x_{j} \in \mathcal{X}$. Replacing $x_{i}$ and $x_{j}$ by $2 x$ and $2 y$ in (2.1), respectively, we get

$$
2 L(x+2 y)+2 L(2 x+y)+L(2 x)+L(2 y)=4 L(2 x+2 y)
$$

for all $x, y \in \mathcal{X}$. Letting $y=0$ in $(2.2)$, we have

$$
L(2 x)=2 L(x)
$$

for all $x \in \mathcal{X}$. It follows from (2.2) and (2.3) that

$$
L(x+2 y)+L(2 x+y)+L(x)+L(y)=4 L(x+y)
$$

for all $x, y \in \mathcal{X}$. Replacing $x$ by $x-y$ in (2.4), we get

$$
L(x+y)+L(2 x-y)+L(x-y)+L(y)=4 L(x)
$$

for all $x, y \in \mathcal{X}$. By putting $x=0$ in $(2.5)$, we have $L(-y)=-L(y)$ for all $y \in \mathcal{X}$. So, $L$ is an odd mapping. Replacing $y$ by $-y$ in (2.5) and using the oddness of $L$, we get

$$
L(x-y)+L(2 x+y)+L(x+y)-L(y)=4 L(x)
$$


for all $x, y \in \mathcal{X}$. Replacing $x$ and $y$ by $y$ and $x$ in (2.6), respectively, and using the oddness of $f$, we get

$$
-L(x-y)+L(x+2 y)+L(x+y)-L(x)=4 L(y)
$$

for all $x, y \in \mathcal{X}$. Adding (2.6) and (2.7), we have

$$
L(2 x+y)+L(x+2 y)+2 L(x+y)=5 L(y)+5 L(x)
$$

for all $x, y \in \mathcal{X}$. Using (2.4) and (2.8), we conclude that $L(x+y)=L(x)+L(y)$ for all $x, y \in \mathcal{X}$. Therefore, $L$ is additive.

We recall that a subadditive mapping is a mapping $\varphi: A \rightarrow B$, having a domain $A$ and a codomain $(B, \leq)$ that are both closed under addition, with the following property:

$$
\varphi(x+y) \leq \varphi(x)+\varphi(y)
$$

for all $x, y \in X$. Now we say that a mapping $\varphi: X \rightarrow Y$ is contractively subadditive if there exists a constant $L$ with $0<L<1$ such that

$$
\varphi(x+y) \leq L[\varphi(x)+\varphi(y)]
$$

for all $x, y \in X$. Therefore $\varphi$ satisfies the following properties $\varphi(\lambda x) \leq \lambda L \varphi(x)$ and so $\varphi\left(\lambda^{n} x\right) \leq(\lambda L)^{n} \varphi(x)$ for all $x \in X$ and all positive integer $\lambda \geq 2$.

Similarly, we say that a mapping $\varphi: A \rightarrow B$ is expansively superadditive if there exists a constant $L$ with $0<L<1$ such that

$$
\varphi(x+y) \geq \frac{1}{L}[\varphi(x)+\varphi(y)]
$$

for all $x, y \in X$. Therefore $\varphi$ satisfies the following properties $\varphi(x) \leq \frac{L}{\lambda} \varphi(\lambda x)$ and so $\varphi\left(\frac{x}{\lambda^{n}}\right) \leq\left(\frac{L}{\lambda}\right)^{n} \varphi(x)$ for all $x \in X$ and all positive integer $\lambda \geq 2$.

Throughout this paper, let $X$ be a linear space over $\mathbb{K}$ and $Y$ is a $(\beta, p)$ Banach space with $p$-norm $\|\cdot\|$. Let $K$ be the modulus of concavity of $\|\cdot\|$. For convenience, we use the following abbreviation for a given function $f: X \rightarrow Y$ :

$$
D f\left(x_{1}, \ldots, x_{n}\right)=2 \sum_{j=1}^{n} f\left(\frac{x_{j}}{2}+\sum_{i=1, i \neq j}^{n} x_{i}\right)+\sum_{j=1}^{n} f\left(x_{j}\right)-2 n f\left(\sum_{j=1}^{n} x_{j}\right)
$$

for all $x_{1}, \ldots, x_{n} \in X$.

Now, we prove the generalized Hyers-Ulam-Rassias stability of CauchyJensen type additive mapping on $(\beta, p)$-Banach spaces for the functional equation $\operatorname{Df}\left(x_{1}, \ldots, x_{n}\right)=0$.

We apply the following theorem:

Theorem 2.2 (Banach). Let $(X, d)$ be a complete metric space and $T: X \rightarrow X$ a contraction, i.e., there exists $\alpha \in[0,1)$ such that

$$
d(T x, T y) \leq \alpha d(x, y), \quad \forall x, y \in X .
$$


Then there exists a unique $a \in X$ such that $T a=a$. Moreover, $a=\lim _{n \rightarrow \infty} T^{n} x$, and

$$
d(a, x) \leq \frac{1}{1-\alpha} d(x, T x) \quad \text { for any } x \in X .
$$

Theorem 2.3. Let $f: X \rightarrow Y$ be a mapping satisfying $f(0)=0$ for which there exists a control function $\varphi: \underbrace{X \times \cdots \times X}_{n \text { times }} \rightarrow(0, \infty)$ such that

$$
\begin{aligned}
& \lim _{k \rightarrow \infty} \frac{1}{2^{k}} \varphi\left(2^{k} x_{1}, \ldots, 2^{k} x_{n}\right)=0, \\
& \left\|D f\left(x_{1}, \ldots, x_{n}\right)\right\| \leq \varphi\left(x_{1}, \ldots, x_{n}\right),
\end{aligned}
$$

for all $x_{1}, \ldots, x_{n} \in X$. If the mapping $\varphi$ is contractively subadditive with a constant $L$ satisfying $2^{(1-\beta)} L<1$, then there exists a unique additive mapping $A: X \rightarrow Y$ such that

$$
\|f(x)-A(x)\| \leq \frac{2 L}{\sqrt[p]{2^{\beta p}-(2 L)^{p}}} \varphi(0, \ldots, 0, \underbrace{x}_{j \text { th }}, 0, \ldots, 0)
$$

for all $x \in X$.

Proof. For convenience, set

$$
\varphi_{j}(x):=\varphi(0, \ldots, 0, \underbrace{x}_{j \text { th }}, 0, \ldots, 0)
$$

for all $x \in X$ and all $1 \leq j \leq n$. Consider the set

$$
\mathcal{M}:=\left\{g: X \rightarrow Y, \quad \sup _{x \in X} \frac{\|g(x)-f(x)\|^{p}}{\varphi_{j}^{p}(x)}<\infty\right\}
$$

and introduce the metric on $\mathcal{M}$ :

$$
d(g, h)=\sup _{x \in X} \frac{\|g(x)-h(x)\|^{p}}{\varphi_{j}^{p}(x)} .
$$

Then $(\mathcal{M}, d)$ is complete. Now we consider the linear mapping $J: \mathcal{M} \rightarrow \mathcal{M}$ such that $J g(x):=\frac{1}{2} g(2 x)$ for all $x \in X$. For any $g, h \in \mathcal{M}$, we have

$$
\begin{aligned}
d(g, h)<\mathcal{C} & \Longrightarrow \frac{\|g(x)-h(x)\|^{p}}{\varphi_{j}^{p}(x)} \leq \mathcal{C}, \quad \forall x \in X \\
& \Longrightarrow \frac{\left\|\frac{1}{2} g(2 x)-\frac{1}{2} h(2 x)\right\|^{p}}{\varphi_{j}^{p}(2 x)} \leq \frac{1}{2^{\beta p}} \mathcal{C} \\
& \Longrightarrow \frac{\left\|\frac{1}{2} g(2 x)-\frac{1}{2} h(2 x)\right\|^{p}}{\varphi_{j}^{p}(x)} \leq\left(2^{1-\beta} L\right)^{p} \mathcal{C} \\
& \Longrightarrow d(J g, J h) \leq\left(2^{1-\beta} L\right)^{p} \mathcal{C} .
\end{aligned}
$$


Therefore, we see that

$$
d(J g, J h) \leq\left(2^{1-\beta} L\right)^{p} d(g, h), \quad \forall g, h \in \mathcal{M} .
$$

This means $J$ is a strictly contractive self-mapping of $\mathcal{M}$, with the Lipschitz constant $\left(2^{1-\beta}\right)^{p} L$.

Letting $x_{j}=2 x$ and for each $1 \leq k \leq n$ with $k \neq j, x_{k}=0$ in (2.10), we get

$$
\frac{\|2 f(x)-f(2 x)\|^{p}}{\varphi_{j}^{p}(2 x)} \leq 1
$$

for all $x \in X$. So

$$
\frac{\left\|f(x)-\frac{1}{2} f(2 x)\right\|^{p}}{\varphi_{j}^{p}(x)} \leq\left(2^{1-\beta} L\right)^{p}
$$

for all $x \in X$. Hence $d(f, J f) \leq\left(2^{1-\beta} L\right)^{p}$.

By Theorem 2.2, there exists a unique mapping $A: X \rightarrow Y$ such that

$$
A(2 x)=2 A(x)
$$

for all $x \in X$, i.e., $A$ is a unique fixed point of $J$. Moreover,

$$
A(x)=\lim _{m \rightarrow \infty} \frac{1}{2^{m}} f\left(2^{m} x\right)
$$

for all $x \in X$. So, we can conclude that $d(f, A) \leq \frac{1}{1-\left(2^{1-\beta} L\right)^{p}} d(f, J f)$, which implies the inequality

$$
d(f, A) \leq \frac{(2 L)^{p}}{2^{\beta p}-(2 L)^{p}} .
$$

This implies that the inequality (2.11) holds.

It follows from $(2.9),(2.10)$ and $(2.14)$ that

$$
\begin{aligned}
& \left\|2 \sum_{j=1}^{n} A\left(\frac{x_{j}}{2}+\sum_{i=1, i \neq j}^{n} x_{i}\right)+\sum_{j=1}^{n} A\left(x_{j}\right)-2 n A\left(\sum_{j=1}^{n} x_{j}\right)\right\| \\
= & \lim _{m \rightarrow \infty} \frac{1}{2^{m \beta}}\left\|2 \sum_{j=1}^{n} f\left(2^{m-1} x_{j}+\sum_{i=1, i \neq j}^{n} 2^{m} x_{i}\right)+\sum_{i=1}^{n} f\left(2^{m} x_{i}\right)-2 n f\left(\sum_{i=1}^{n} 2^{m} x_{i}\right)\right\| \\
\leq & \lim _{m \rightarrow \infty} \frac{1}{2^{m \beta}} \varphi\left(2^{m} x_{1}, \ldots, 2^{m} x_{n}\right) \\
\leq & \lim _{m \rightarrow \infty}\left(2^{1-\beta} L\right)^{m} \varphi\left(x_{1}, \ldots, x_{n}\right)
\end{aligned}
$$

for all $x_{1}, \ldots, x_{n} \in X$. So

$$
2 \sum_{j=1}^{n} A\left(\frac{x_{j}}{2}+\sum_{i=1, i \neq j}^{n} x_{i}\right)+\sum_{i=1}^{n} A\left(x_{i}\right)=2 n A\left(\sum_{i=1}^{n} x_{i}\right)
$$

for all $x_{1}, \ldots, x_{n} \in X$. By Lemma 2.1, the mapping $A: X \rightarrow X$ is Cauchy additive, i.e., $A(x+y)=A(x)+A(y)$ for all $x, y \in X$. 
Theorem 2.4. Let $f: X \rightarrow Y$ be a mapping satisfying $f(0)=0$ for which there exists a control function $\varphi: \underbrace{X \times \cdots \times X}_{n \text { times }} \rightarrow(0, \infty)$ such that

$$
\begin{aligned}
& \lim _{k \rightarrow \infty} 2^{k} \varphi\left(\frac{x_{1}}{2^{k}}, \ldots, \frac{x_{n}}{2^{k}}\right)=0, \\
& \left\|D f\left(x_{1}, \ldots, x_{n}\right)\right\| \leq \varphi\left(x_{1}, \ldots, x_{n}\right),
\end{aligned}
$$

for all $x_{1}, \ldots, x_{n} \in X$. If the mapping $\varphi$ is expansively superadditive with a constant $L$ satisfying $2^{(\beta-1)} L<1$. Then there exists a unique additive mapping $A: X \rightarrow Y$ such that

$$
\|f(x)-A(x)\| \leq \frac{2}{\sqrt[p]{2^{p}-\left(2^{\beta} L\right)^{p}}} \varphi(0, \ldots, 0, \underbrace{x}_{j \text { th }}, 0, \ldots, 0)
$$

for all $x \in X$.

Proof. Similar to the proof of Theorem 2.3, we consider the linear mapping $J: \mathcal{M} \rightarrow \mathcal{M}$ such that $J g(x):=2 g\left(\frac{x}{2}\right)$ for all $x \in X$. For any $g, h \in \mathcal{M}$, we have

$$
\begin{aligned}
d(g, h)<\mathcal{C} & \Longrightarrow \frac{\|g(x)-h(x)\|^{p}}{\varphi_{j}^{p}(x)} \leq \mathcal{C}, \quad \forall x \in X \\
& \Longrightarrow \frac{\left\|2 g\left(\frac{x}{2}\right)-2 h\left(\frac{x}{2}\right)\right\|^{p}}{\varphi_{j}^{p}\left(\frac{x}{2}\right)} \leq 2^{\beta p} \mathcal{C} \\
& \Longrightarrow \frac{\left\|2 g\left(\frac{x}{2}\right)-2 h\left(\frac{x}{2}\right)\right\|^{p}}{\varphi_{j}^{p}(x)} \leq\left(2^{\beta-1} L\right)^{p} \mathcal{C} \\
& \Longrightarrow d(J g, J h) \leq\left(2^{1-\beta} L\right)^{p} \mathcal{C} .
\end{aligned}
$$

Therefore, we see that

$$
d(J g, J h) \leq\left(2^{\beta-1} L\right)^{p} d(g, h), \quad \forall g, h \in \mathcal{M} .
$$

This means $J$ is a strictly contractive self-mapping of $\mathcal{M}$, with the Lipschitz constant $\left(2^{1-\beta} L\right)^{p}$.

Letting $x_{j}=x$ and for each $1 \leq k \leq n$ with $k \neq j, x_{k}=0$ in (2.16), we get

$$
\frac{\left\|f(x)-2 f\left(\frac{x}{2}\right)\right\|^{p}}{\varphi_{j}^{p}(x)} \leq 1
$$

for all $x \in X$. Hence $d(f, J f) \leq 1$.

By Theorem 2.2, there exists a unique mapping $A: X \rightarrow Y$ such that

$$
A\left(\frac{x}{2}\right)=\frac{1}{2} A(x)
$$

for all $x \in X$, i.e., $A$ is a unique fixed point of $J$. Moreover,

$$
A(x)=\lim _{m \rightarrow \infty} 2^{m} f\left(\frac{x}{2^{m}}\right)
$$


for all $x \in X$. So, we can conclude that $d(f, A) \leq \frac{1}{1-\left(2^{\beta-1} L\right)^{p}} d(f, J f)$, which implies the inequality

$$
d(f, A) \leq \frac{2^{p}}{2^{p}-\left(2^{\beta} L\right)^{p}} .
$$

This implies that the inequality (2.17) holds.

The rest of the proof is similar to the proof of Theorem 2.3.

\section{References}

[1] J. Aczél and J. Dhombres, Functional Equations in Several Variables, Cambridge University Press, 1989.

[2] T. Aoki, On the stability of the linear transformation in Banach spaces, J. Math. Soc. Japan 2 (1950), 64-66.

[3] Y. Benyamini and J. Lindenstrauss, Geometric Nonlinear Functional Analysis. Vol. 1, Colloq. Publ., vol. 48, Amer. Math. Soc., Providence, RI, 2000.

[4] C. Borelli and G. L. Forti, On a general Hyers-Ulam stability result, Internat. J. Math. Math. Sci. 18 (1995), no. 2, 229-236.

[5] D. G. Bourgin, Classes of transformations and bordering transformations, Bull. Amer. Math. Soc. 57 (1951), 223-237.

[6] L. Cădariu and V. Radu, Fixed points and the stability of Jensen's functional equation, J. Inequal. Pure Appl. Math. 4 (2003), no. 1, Article 4, 7 pp.

[7] — Fixed points and the stability of quadratic functional equations, An. Univ. Timişoara, Ser. Mat.-Inform. 41 (2003), no. 1, 25-48.

[8] - On the stability of the Cauchy functional equation: a fixed point approach, Iteration theory (ECIT '02), 43-52, Grazer Math. Ber., 346, Karl-Franzens-Univ. Graz, Graz, 2004.

[9] S. Czerwik, The stability of the quadratic functional equation, In: Th. M. Rassias, J. Tabor (Eds.), Stability of Mappings of Hyers-Ulam Type, pp. 81-91, Hadronic Press, Florida, 1994.

[10] P. Găvruta, A generalization of the Hyers-Ulam-Rassias stability of approximately additive mappings, J. Math. Anal. Appl. 184 (1994), no. 3, 431-436.

[11] P. Găvruta and L. Găvruta, A new method for the generalized Hyers-Ulam-Rassias stability, Int. J. Nonlinear Anal. Appl. 1 (2010), no. 2, 11-18.

[12] D. H. Hyers, On the stability of the linear functional equation, Proc. Nat. Acad. Sci. U.S.A. 27 (1941), 222-224.

[13] D. H. Hyers, G. Isac, and Th. M. Rassias, Stability of Functional Equations in Several Variables, Birkhauser, Boston,Basel, Berlin, 1998.

[14] K. Jun and Y. Lee, On the Hyers-Ulam-Rassias stability of a Pexiderized quadratic inequality, Math. Inequal. Appl. 4 (2001), no. 1, 93-118.

[15] S.-M. Jung, Hyers-Ulam-Rassias Stability of Functional Equations in Nonlinear Analysis, Springer, New York, 2011.

[16] S.-M. Jung and J. M. Rassias, A fixed point approach to the stability of a functional equation of the spiral of Theodorus, Fixed Point Theory Appl. 2008 (2008), Art. ID 945010, 7 pp.

[17] Pl. Kannappan,Functional Equations and Inequalities with Applications, Springer, New York, 2009.

[18] G. H. Kim, On the stability of the quadratic mapping in normed spaces, Int. J. Math. Math. Sci. 25 (2001), no. 4, 217-229.

[19] F. Moradlou, Additive functional inequalities and derivations on Hilbert $C^{*}$-modules, Glasg. Math. J. 55 (2013), 341-348. 
[20] F. Moradlou, A. Najati, and H. Vaezi, Stability of homomorphisms and derivations on $C^{*}$-ternary rings associated to an Euler-Lagrange type additive mapping, Results Math. 55 (2009), no. 3-4, 469-486.

[21] F. Moradlou, H. Vaezi, and G. Z. Eskandani, Hyers-Ulam-Rassias stability of a quadratic and additive functional equation in quasi-Banach spaces, Mediterr. J. Math. 6 (2009), no. 2, 233-248.

[22] F. Moradlou, H. Vaezi, and C. Park, Fixed points and stability of an additive functional equation of $n$-Apollonius type in $C^{*}$-algebras, Abstr. Appl. Anal. 2008 (2008), Art. ID 672618, $13 \mathrm{pp}$.

[23] _ Approximate Euler-Lagrange-Jensen type additive mapping in multi-Banach spaces, A Fixed Point Approach, Commun. Korean Math. Soc. 28 (2013), no. 2, 319333.

[24] P. M. Pardalos, P. G. Georgiev, and H. M. Srivastava (eds.), Nonlinear Analysis, Stability, Approximation and Inequalities. In honor of Themistocles M. Rassias on the occasion of his 60th birthday, Springer, New York, 2012.

[25] C. Park, On the stability of the linear mapping in Banach modules, J. Math. Anal. Appl. $\mathbf{2 7 5}$ (2002), no. 2, 711-720.

[26] - On an approximate automorphism on a $C^{*}$-algebra, Proc. Amer. Math. Soc. 132 (2004), no. 6, 1739-1745.

[27] _ Homomorphisms between Poisson JC*-algebras, Bull. Braz. Math. Soc. 36 (2005), no. 1, 79-97.

[28] C. Park and Th. M. Rassias, Hyers-Ulam stability of a generalized Apollonius type quadratic mapping, J. Math. Anal. Appl. 322 (2006), no. 1, 371-381.

[29] - Homomorphisms in $C^{*}$-ternary algebras and JB*-triples, J. Math. Anal. Appl. 337 (2008), no. 1, 13-20.

[30] V. Radu, The fixed point alternative and stability of functional equations, Fixed Point Theory IV(1) (2003), no. 1, 91-96.

[31] J. M. Rassias, On approximation of approximately linear mappings by linear mappings, J. Funct. Anal. 46 (1982), no. 1, 126-130.

[32] _ On approximation of approximately linear mappings by linear mappings, Bull. Sci. Math. 108 (1984), no. 4, 445-446.

[33] _ Solution of a problem of Ulam, J. Approx. Theory 57 (1989), no. 3, 268-273.

[34] _ Alternative contraction principle and Ulam stability problem, Math. Sci. Res. J. 9 (2005), no. 7, 190-199.

[35] J. M. Rassias and H. M. Kim, Generalized Hyers-Ulam stability for general additive functional equations in quasi- $\beta$-normed spaces, J. Math. Anal. Appl. 356 (2009), no. 1, 302-309.

[36] Th. M. Rassias, On the stability of the linear mapping in Banach spaces, Proc. Amer. Math. Soc. 72 (1978), no. 2, 297-300.

[37] _ The problem of S. M. Ulam for approximately multiplicative mappings, J. Math. Anal. Appl. 246 (2000), no. 2, 352-378.

[38] _ On the stability of functional equations in Banach spaces, J. Math. Anal. Appl. 251 (2000), no. 1, 264-284.

[39] Th. M. Rassias and J. Brzdek (eds.), Functional Equations in Mathematical Analysis, Springer, New York, 2012.

[40] S. Rolewicz, Metric Linear Spaces, PWN-Polish Sci. Publ., Warszawa, Reidel, Dordrecht, 1984.

[41] F. Skof, Local properties and approximations of operators, Rend. Sem. Mat. Fis. Milano 53 (1983), 113-129.

[42] S. M. Ulam, A Collection of the Mathematical Problems, Interscience Publ. New York, 1960. 
Fridoun MoradLOU

Department of Mathematics

SAHAND University of TeChNOLOGY

TABRIZ, IRAN

E-mail address: moradlou@sut.ac.ir, fridoun.moradlou@gmail.com

Themistocles M. Rassias

Department of Mathematics

National Technical University of Athens

Zografou Campus, 15780 Athens, Greece

E-mail address: trassias@math.ntua.gr 